\title{
MECANISMOS CITOLÓGICOS INVOLUCRADOS EN LA PRODUCCIÓN DE GAMETOS MASCULINOS NO REDUCIDOS EN INDIVIDUOS DIPLOIDES DE TURNERA SIDOIDES SUBSP. CARNEA (PASSIFLORACEAE)
}

\author{
IVANA EVELIN KOVALSKY ${ }^{1,2}$, AVELIANO FERNÁNDEZ ${ }^{1}$ y VIVIANA G. SOLÍS NEFFA ${ }^{1,2}$
}

\begin{abstract}
Summary: Cytological mechanisms involved in the unreduced male gametes production in diploid individuals of Turnera sidoides subsp. carnea (Passifloraceae). Turnera sidoides $(x=7)$ is an autopolyploid complex of outcrossing perennial herbs that includes five subspecies, with ploidy levels from diploid $(2 \mathrm{n}$ $=2 x=14)$ to octoploid $(2 n=8 x=56)$. Previous studies have revealed the production of unreduced male gametes $(2 n$ and $4 n)$ in diploid populations of this complex, suggesting that sexual polyploidyzation would be the main mechanism involved in the origin of neopoliploids of $T$. sidoides. However, to date, there is a lack of data about the cytological mechanisms involved in the origin of such unreduced gametes. Thus, in this paper we analyze the meiotic behaviour and pollen viability of unreduced gametes producers from a diploid population of $T$. sidoides subsp. carnea. The results obtained showed that meiotic behavior was mostly regular; however some irregularities such as parallel spindles, fused spindles and nuclear restitution were also observed. Pollen viability varied from $44.75 \%$ to $95.82 \%$. Overall, our results suggest that nuclear restitution at both the first and the second meiotic division would be the cytological mechanisms involved in the production of $2 n$ male gametes. Such mechanisms would have important consequences in the establishments of neopolyploids in natural populations.
\end{abstract}

Key words: Turnera sidoides, poliploidy, unreduced gametes, meiosis, pollen viability.

Resumen: Turnera sidoides $(x=7)$ es un complejo autopoliploide de hierbas alógamas perennes que incluye cinco subespecies, con niveles de ploidía desde diploide $(2 n=2 x=14)$ hasta octoploide $(2 n=$ $8 x=56$ ). Estudios previos aportaron evidencias de la producción de gametos masculinos no reducidos ( $2 n$ y $4 n$ ) en poblaciones diploides del complejo, sugiriendo que los poliploides de $T$. sidoides habrían surgido por poliploidización sexual. Sin embargo, hasta el momento se desconocen los mecanismos citológicos involucrados en la producción de dichos gametos. Por lo tanto, en este trabajo se analiza el comportamiento meiótico y la viabilidad del polen de individuos productores de gametos no reducidos de una población diploide de $T$. sidoides subsp. carnea. Los resultados obtenidos evidenciaron que la meiosis es generalmente regular, aunque también se observaron algunas irregularidades como husos paralelos, husos fusionados y núcleos de restitución. La viabilidad del polen varió entre $44,75 \%$ y $95,82 \%$. Todos estos resultados sugieren que la restitución nuclear tanto en la primera división como en la segunda división meiótica serían los mecanismos involucrados en la producción de gametos masculinos no reducidos en el complejo. Estos mecanismos tendrían importantes consecuencias en el establecimiento de neopoliploides en poblaciones naturales de $T$. sidoides.

Palabras clave: Turnera sidoides, poliploidía, gametos no reducidos, meiosis, viabilidad del polen.

${ }^{1}$ Laboratorio de Citogenética y Evolución Vegetal. Instituto de Botánica del Nordeste (UNNE-CONICET). C.C. 209, 3400, Corrientes, Argentina.

${ }^{2}$ Facultad de Ciencias Exactas y Naturales y Agrimensura (UNNE).

e-mail: evelinkov@yahoo.com.ar 


\section{INTRODUCCIÓN}

La poliploidía constituye uno de los principales mecanismos de evolución y diversificación en plantas. Diferentes estimaciones sugieren que el $95 \%$ de las Pteridófitas y el $70 \%$ de las Angiospermas han experimentado uno o más episodios de poliploidización (Stebbins, 1971; Grant, 1989). Entre ellas, algunas de las especies cultivadas, así como numerosas especies hortícolas y forrajeras son de origen poliploide (Ramanna \& Jacobsen, 2003).

Dada la importancia de la poliploidía, numerosos estudios tendientes a examinar su significado evolutivo estuvieron orientados a la comprensión de los procesos involucrados en el origen de los poliploides y de las condiciones que favorecen su establecimiento y persistencia (Thompson \& Lumaret, 1992; Bretagnolle \& Thompson, 1995; Ramsey \& Schemske, 1998). En relación al origen, se ha demostrado que los poliploides se pueden formar por duplicación cromosómica somática en los tejidos meristemáticos del esporofito, cigotos o embriones, o por poliploidización sexual. Este último, es considerado el principal mecanismo de origen de los poliploides en plantas, y consiste en fertilizaciones que involucran a gametos no reducidos, es decir aquellos gametos que, como resultado de irregularidades en la gametogénesis, poseen el número de cromosomas somático (Karpechenko, 1927; Darlington, 1937, 1965; Harlan \& De Wet, 1975; De Wet, 1980; Bretagnolle \& Thompson, 1995; Ramsey \& Schemske, 1998).

Estudios experimentales llevados a cabo como parte de programas de mejoramiento genético de diversos cultivos han contribuido en gran medida al esclarecimiento de los mecanismos citológicos que conducen a la formación de gametos no reducidos, así como de los factores genéticos y ambientales que influyen en su producción (Hermsen, 1984; Veilleux, 1985; Singh et al., 1990; Bretagnolle \& Thompson, 1995; Bingham et al., 1991; Ramanna, 1992; Ramanna \& Jacobsen, 2003; Lyrene et al., 2003; d'Erfurth et al., 2008; De Storme \& Geelen, 2011; Mason et al., 2011). Los mecanismos involucrados en la formación de gametos no reducidos, operan en la microsporogénesis y/o la megasporogénesis (Harlan \& De Wet, 1975; Jackson \& Casey, 1980; Hermsem, 1984; Kaul \& Murthy, 1985; Veilleux, 1985; Andreuzza \& Siddiqi,
2008; De Storme \& Geelen, 2011). Los estudios genéticos, citológicos e inmunocitoquímicos en especies modelo, han permitido la identificación de genes que regulan los eventos meióticos, incluyendo la recombinación (Atchenson et al., 1987; Dernburg et al., 1998; Grelon et al., 2001), el ensamblaje del huso (Musacchio \& Hardick, 2002; Lew \& Burket, 2003), la orientación del cinetocoro y la segregación cromosómica (Watanabe \& Nurse, 1999; Toth et al., 2000; Watanabe \& Yokobayashi, 2005) y, la citocinesis (Nacry et al., 2000; Giansanti et al., 2004). La mutación de alguno de dichos genes puede alterar la meiosis, afectando la fertilidad gamética $y / 0$ conduciendo a la formación de núcleos de restitución (i.e. núcleos con el número no reducido de cromosomas). Estos mecanismos, así como las duplicaciones pre y postmeióticas, resultan en la formación de gametos no reducidos (Ramanna, 1979; Veilleux, 1985; Bretagnolle \& Thompson, 1995; Qu \& Vorsa, 1999; Ramanna \& Jacobsen, 2003; d'Erfurth et al., 2008).

Estudios citológicos y genéticos realizados en diferentes especies han demostrado la ocurrencia de varios tipos de restitución nuclear, tales como la restitución en la primera (RPD) y en la segunda división meiótica (RSD) y un mecanismo detectado en híbridos de Lilium denominado restitución meiótica indeterminada (RMI) (Lim et al., 2001; Barba Gonzalez et al., 2005; Zhou et al., 2008; Khan et al., 2010), aunque las más comunes son la RPD y RSD. Dichos tipos de restitución nuclear difieren tanto citológicamente como en sus consecuencias genéticas.

Durante el proceso normal de la meiosis, en la primera división se separan los cromosomas homólogos y en la segunda se separan las cromátidas hermanas de cada cromosoma. En la RPD, no se produce el apareamiento y/o la separación de los cromosomas homólogos en la primera división meiótica; mientras que la segunda división ocurre normalmente. En la RSD, el apareamiento y separación de los cromosomas homólogos ocurre normalmente durante la primera división meiótica, mientras que en la segunda división las cromátidas hermanas se separan durante la Anafase II pero posteriormente el núcleo restituye (Bretagnolle \& Thompson, 1995). Finalmente, en la RMI, durante la primera división meiótica todos los univalentes se dividen ecuacionalmente mientras que los bivalentes lo hacen reduccionalmente. Asimismo, 


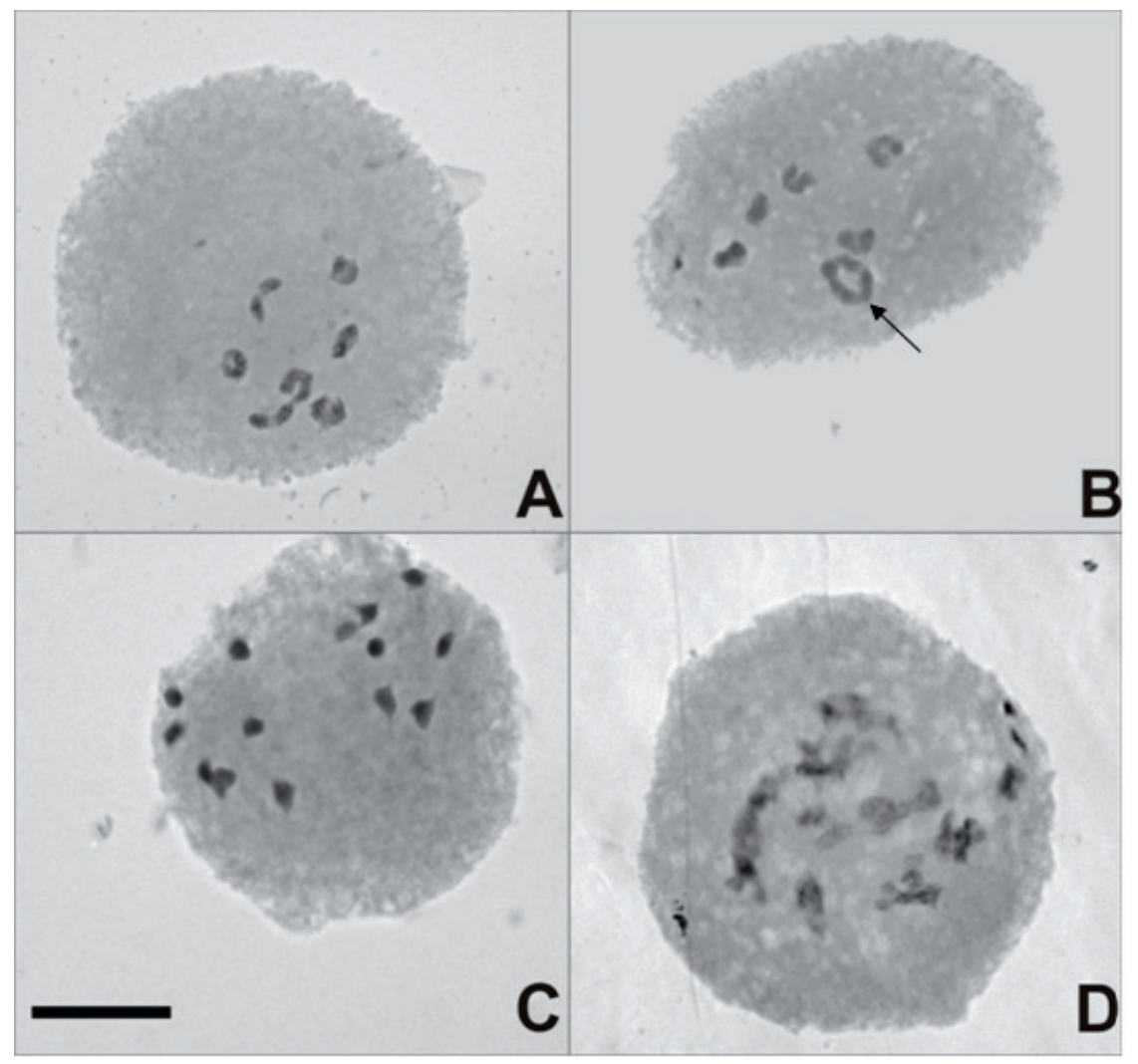

Fig. 1. Cromosomas meióticos de individuos diploides productores de gametos no reducidos de Turnera sidoides subsp. carnea. A-B: Metafase I. A: 7II. B: 5 II + 1 IV (flecha). C: Profase II normal. D: Núcleo de restitución de la primera división. Escala: $5 \mu \mathrm{m}$.

debido a las diferencias en la segregación de los cromosomas, los efectos en la heterocigosis de estos tres mecanismos también son diferentes (Ramanna, 1983; Bretagnolle \& Thompson, 1995; Lim et al., 2001; Barba Gonzalez et al., 2008).

Turnera sidoides $\mathrm{L} .(\mathrm{x}=7)$ es un excelente modelo biológico para investigar los mecanismos de origen y establecimiento de los poliploides. Este complejo autopoliploide de hierbas alógamas perennes incluye cinco subespecies (Arbo, 1985), con niveles de ploidía desde diploide, $2 \mathrm{n}=2 \mathrm{x}=$ 14, hasta octoploide, $2 \mathrm{n}=8 \mathrm{x}=56$ (Fernández, 1987; Solís Neffa \& Fernández, 2001; Solís Neffa et al., 2004; Elías et al., 2011; Kovalsky \& Solís Neffa, 2012). Se ha sugerido que los poliploides del complejo habrían surgido por poliploidización sexual (Solís Neffa, 2000; Panseri et al., 2008; Kovalsky \& Solís Neffa, 2012).
En este sentido, estudios previos llevados a cabo en poblaciones diploides experimentales, aportaron evidencias de la producción de gametos masculinos no reducidos en T. sidoides (Panseri et al., 2008). Por otra parte, un estudio exhaustivo llevado a cabo en poblaciones naturales de este complejo reveló una gran variación en la frecuencia de producción de gametos no reducidos $(2 n)$ y duplicados $(4 n)$. Dichas investigaciones también demostraron que las plantas de una misma población difieren en su capacidad de producir gametos no reducidos y que la frecuencia de producción de este tipo de gametos es muy variable aún dentro de las flores de una misma planta. Asimismo, las condiciones ambientales también influirían en la formación de gametos $2 n$ (Kovalsky \& Solís Neffa, 2012; Kovalsky, 2012). Sin embargo, hasta el momento se desconocen los mecanismos de origen de los gametos no reducidos en T. sidoides. 
Sobre la base de estos antecedentes y a fin de contribuir a la comprensión de los mecanismos citológicos involucrados en la producción de gametos masculinos no reducidos en el complejo Turnera sidoides, en este trabajo se analiza el comportamiento meiótico y la viabilidad del polen de individuos productores de gametos $2 n$ y $4 n$ de una población diploide de T. sidoides subsp. carnea (Cambess.) Arbo.

\section{Materiales y Métodos}

Se analizó el comportamiento meiótico y la viabilidad del polen de 17 individuos diploides productores de gametos no reducidos provenientes de una población de Turnera sidoides subsp. carnea, localizada en la ruta 123 y el río Miriñay

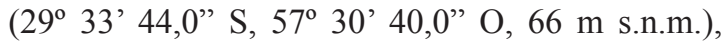
en el Departamento de Mercedes, Corrientes (Argentina). El ejemplar testigo (Solís Neffa \& Seijo 960) se encuentra depositado en el Herbario del Instituto de Botánica del Nordeste (CTES). Se seleccionó esta población, debido a que en estudios previos se detectó una alta producción de gametos masculinos no reducidos (Panseri et al., 2008; Kovalsky \& Solís Neffa, 2012). La distribución espacial de las plantas productoras de gametos masculinos no reducidos fue georeferenciada $y$, algunas de ellas, fueron transportadas a Corrientes donde se cultivaron bajo condiciones controladas en el invernáculo del mismo instituto.

El estudio del comportamiento meiótico se realizó a partir de botones florales obtenidos de plantas productoras de gametos no reducidos creciendo en condiciones naturales y de invernáculo. Los botones florales de tamaño adecuado fueron fijados en alcohol etílico absoluto: ácido láctico (5: 1) (Fernández, 1973) durante $12 \mathrm{~h} \mathrm{a} 4^{\circ} \mathrm{C}$ y conservados en etanol $70 \%$ a $4^{\circ} \mathrm{C}$. Los preparados se obtuvieron por aplastado de las anteras en orceína acética y se hicieron permanentes por el método de congelación con $\mathrm{CO}_{2}$ (Bowen, 1956) utilizándose Euparal como medio de montaje.

La viabilidad de los granos de polen fue estimada mediante la técnica de coloración con carmínglicerina (1:1) (Pittenger \& Frolik, 1951). Las anteras fueron colectadas durante las primeras horas de la mañana y aplastadas en una gota de colorante. Se consideraron viables los granos teñidos totalmente. Se contaron al menos 300 granos por flor $\mathrm{y}$, en lo posible, no menos de 4 flores por planta.

\section{Resultados}

El análisis de 849 células madres del polen mostró que las células en Diacinesis-MI presentaron generalmente 7II $(81,76 \%$, Fig. 1A), aunque también se observaron 1-2 IV (3,86\%, Fig. 1B), 1-2 I $(1,10 \%)$, así como células con bivalentes fuera de placa (11,60\%). En A-T I y II, la segregación de los cromosomas fue generalmente normal (Fig. 1 C), no obstante también se observó la segregación precoz y desbalanceada de los cromosomas (2,65\%), además de células con núcleos de restitución (1,65\%, Fig 1D). En MII se observaron puentes residuales, husos paralelos y husos fusionados $(7,24 \% ; 30,43 \%$ y $10,14 \%$, respectivamente), mientras que en TII se observaron células con tres núcleos en lugar de 4 (7,09\%). Asimismo, a partir del análisis de 190.070 granos de polen se comprobó que el promedio de viabilidad del polen fue de $87,70 \%$, variando entre $44,74 \%$ y $95,82 \%$, entre los individuos analizados.

\section{Discusión}

Los resultados obtenidos en la población diploide de T. sidoides subsp. carnea constituyen el primer aporte a la comprensión de los mecanismos citológicos involucrados en la formación de gametos no reducidos en el complejo $T$. sidoides y en el género Turnera.

Dichos resultados evidenciaron que la meiosis en los individuos diploides productores de gametos masculinos no reducidos de T. sidoides subsp. carnea es generalmente regular, aunque el porcentaje de irregularidades meióticas fue mayor al observado en otras poblaciones diploides de $T$. sidoides (Fernández, 1987; Solís Neffa, 2000) y en otras especies diploides de Turnera (Fernández, 1987) no productoras de gametos no reducidos. Por otra parte, el porcentaje de polen inviable en los individuos analizados está de acuerdo con el porcentaje de irregularidades meióticas detectados en la población estudiada, siendo la viabilidad del polen menor a la observada en otras poblaciones diploides de Turnera (Fernández, 1987; Solís Neffa, 2000). 


\section{E. Kovalsky et al. - Mecanismos citológicos de producción de gametos 2n en T. sidoides}

En relación a las irregularidades meióticas, los puentes observados en Anafase I y la presencia de tetravalentes sugieren la existencia de cambios estructurales en los cromosomas de la población estudiada. Asimismo, la presencia de cromosomas rezagados y fuera de placa, explicarían la reducción de la fertilidad gamética observada en los individuos analizados. Finalmente, los husos paralelos, los husos fusionados y los núcleos de restitución observados podrían conducir a la formación de gametos $2 n$ como también fuera sugerido en otras especies (Harlan \& De Wet, 1975; Veilleux, 1985; Bretagnolle \& Thompson, 1995).

En este sentido, se ha propuesto que las aberraciones en la formación del huso meiótico y en la citocinesis constituyen la principal causa de la restitución nuclear en la microsporogénesis en diversas especies (Veilleux, 1985; Conicella et al., 1991; Bretagnolle \& Thompson, 1995; d'Erfurth et al., 2008; Camadro et al., 2008; Xu et al., 2008; Zhang \& Kang, 2010). En Brachiaria, por ejemplo, se encontró que tanto las fallas de la citocinesis en la meiosis I como la total ausencia de citocinesis resultan en la formación de gametos masculinos con cromosomas duplicados. En Pfaffia glomerata (Spreng.) Pedersen y P. tuberosa Spreng. ocurre a menudo una orientación anormal en los ejes de la meiosis, formándose en Metafase II husos paralelos o tripolares los que son responsables de la formación de díadas y tríadas que originan luego granos de polen $2 n$ (Taschetto \& Pagliarini, 2003). Los husos paralelos, y fusionados son también los responsables del origen del polen $2 n$ en Solanum okadae Hawkes \& Hjert. y en Populus tomentosa C. K. Schneid. (Camadro et al., 2008; Zhang \& Kang, 2010). Por otra parte, en haploides e híbridos interespecíficos de trigo, así como en papas diploides, la restitución del núcleo ha sido relacionada con la sinapsis anormal de los cromosomas durante la profase de la primera división meiótica (Koduru \& Rao, 1981; Ramanna, 1983; Kaul \& Murthy, 1985; Xu \& Dong, 1992; Xu \& Joppa, 1995; Lim et al., 2001; Ramanna \& Jacobsen, 2003). En Solanum, dichas anomalías fueron denominadas "mutantes sinápticos" (Jongedijk \& Ramanna, 1988). Estos mutantes se caracterizan por un aumento en la frecuencia de univalentes en Metafase I como resultado de errores en el apareamiento durante la Profase. En algunos casos, los gametos resultantes son estériles debido al número desbalanceado de cromosomas, mientras que en otros casos se pueden formar gametos $2 n$ balanceados y fértiles. Los mutantes sinápticos pueden originar una progenie euploide o aneuploide (Ramanna, 1983; Parrott \& Smith, 1984; Jongedijk, 1985; Parrott \& Hanneman, 1988).

A partir del análisis de las esporadas y de la variación del tamaño del polen, se demostró la presencia de mónadas, díadas y tríadas, así como de polen no reducido y duplicado ("jumbo") en poblaciones experimentales y naturales de $T$. sidoides (Panseri et al., 2008; Kovalsky \& Solís Neffa, 2012). La formación de husos paralelos y fusionados observados en este trabajo, explicarían en parte la formación de las díadas y tríadas, así como de polen $2 n$ en esta especie. Sin embargo, dado que la frecuencia de husos paralelos y fusionados observada en los individuos productores de gametos masculinos no reducidos es mayor que la frecuencia de díadas y tríadas $(0,09-0,12 \%)$ detectada previamente en los mismos individuos de T. sidoides subsp. carnea (Kovalsky \& Solís Neffa, 2012; Kovalsky, 2012) así como en otras subespecies del complejo (Panseri et al., 2008), es probable que en $T$. sidoides la formación de los husos paralelos no siempre conduzca a la formación de gametos no reducidos. La falta de correspondencia entre la frecuencia de este tipo de irregularidades meióticas y la frecuencia de díadas y tríadas también fue observada en Ziziphus jujuba Mill. (Xue et al., 2011). Asimismo, en T. sidoides la formación de núcleos de restitución en la primera división seguida de una división ecuacional y posterior citocinesis en la segunda división, también explicaría la formación de díadas y polen $2 n$; mientras que si la RPD es seguida por la RSD, ambas conducirían a la formación de mónadas y por lo tanto a la formación de polen jumbo. Estos resultados sugieren que tanto la RPD como la RSD estarían involucrados en la producción de los gametos $2 n$ y $4 n$ observados en $T$. sidoides subsp. carnea.

Por otra parte, debido a las diferencias en las consecuencias genéticas de los distintos mecanismos de restitución nuclear, el tipo de mecanismo involucrado en el origen de los gametos no reducidos podría influir en el establecimiento de los neopoliploides de $T$. sidoides originados en poblaciones naturales. En este sentido, se ha sugerido que los gametos no reducidos producidos por RPD contienen niveles equivalentes de 
heterocigosis y de combinaciones epistáticas que ambos progenitores; mientras que los gametos formados por RSD poseen menos heterocigosis y combinaciones epistáticas que los parentales (Mendiburu \& Peloquín, 1977; Bingham, 1980; Ramanna, 1983; Hermsen, 1984; Veilleux, 1985; Werner \& Peloquín, 1991 a, b, c; Bretagnolle \& Thompson, 1995). Aunque en T. sidoides subsp. carnea los gametos no reducidos se originarían tanto por RPD como por RSD, los gametos $2 n$ formados por RPD podrían mantener los niveles de heterocigosis y las combinaciones epistáticas de los progenitores en los neopoliploides, contribuyendo al establecimiento de los mismos en las poblaciones diploides y su posterior expansión. Estudios en curso en poblaciones diploides y en las zonas de contacto diploide-tetraploide permitirán dilucidar cuáles son las consecuencias genéticas y evolutivas de estos mecanismos de restitución en T. sidoides.

\section{Agradecimientos}

Este trabajo fue financiado por proyectos de la Agencia Nacional de Promoción Científica, Tecnológica y de Innovación (ANPCyT- FONCyT, PICT 01-1329 y PICTO 07-90), y de la Secretaría General de Ciencia y Técnica (UNNE, PI-014/07). I.E. Kovalsky es becaria Postdoctoral de CONICET, y V.G. Solís Neffa es miembro de la Carrera del Investigador Científico de CONICET.

\section{Bibliografía}

ANDREUZZA, S. \& I. SIDDIQI. 2008. Spindle positioning, meiotic non reduction, and polyploidy in plants. PLoS Genet. 4: e1000272. doi:10.1371/ journal.pgen. 1000272 .

ARBO, M. M. 1985. Notas taxonómicas sobre Turneráceas americanas. Candollea 40: 175-191.

ATCHENSON, C. L., B. DI DOMENICO, S. FRACKMAN, R. E. ESPOSITO \& R. T. ELDER. 1987. Isolation, DNA sequence, and regulation of meiotic specific eukaryotic recombination gene. Candollea 84: 8035-8039.

BARBA GONZALEZ, R., K. B. LIM, M. S. RAMANNA, R. G. F. VISSER \& J. M. VAN TUYL. 2005. Intergenomic recombination in the $\mathrm{F}_{1}$ hybrids of Oriental Asiatic lily hybrids (Lilium) and its significance for genetic variation in the $\mathrm{BC} 1$ progenies as revealed by GISH and FISH analyses. Genome 48: 884-894.

BARBA GONZALEZ R., K. B. LIM, S. ZHOU, M. S. RAMANNA \& J. VAN TUYL. 2008. Interspecific hybridization, In: Lily: The use of $2 n$ gametes in interspecific Lily hybrids floriculture. Ornamental and Plant Biotechnology Volume V. Global Science Books, UK. 13: 138-145.

BINGHAM, E. T. 1980. Maximizing heterozygosity in autopolyploids. In: LEWIS W. H. (ed.), Polyploidy, biological relevance, pp. 471-490. Plenum Press, New York.

BINGHAM, E. T., R. R. SMITH. \& M. D. CASLER. 1991. Reproduction dynamics of polyploid forage legumes and grasses. In: Proc. Eucarpia Meeting, pp. 7-13. Alghero, Sassari, Italy.

BOWEN, C. 1956. Freezing by liquid carbone dioxide in making slides permanent. Stain Technol. 31: 87-90.

BRETAGNOLLE, F. \& J. D. THOMPSON. 1995. Gametes with the somatic chromosome number: mechanisms of their formation and role in the evolution of autopolyploid plants. New Phytol. 129: $1-22$.

CAMADRO, E. L., S. K. SAFFARANO, J. C. ESPINILLO, M. CASTRO \& P. W. SIMON. 2008. Cytological mechanisms of $2 n$ pollen formation in the wild potato Solanum okadae and pollenpistil relations with the cultivated potato, Solanum tuberosum. Genet. Resour. Crop. Evol. 55: 471-477. doi:10.1007/ s10722-007-9254-1.

CONICELlA, C. A., C. A. BARONE, A. DEL GIUDICE, L. FRUCIANTE \& L. M. MONTI. 1991. Cytological evidence of SDR-FDR mixture in the formation of $2 n$ eggs in a potato diploid clone. Theor. Appl. Genet. 81: 59-63.

DARLINGTON, C. D. 1937. Recent advances in cytology. Blakinston's Son and Co. Inc., Philadelphia.

DARLINGTON, C. D. 1965. Recent advances in cytology. J. and A. Churchill Ltd., London.

D'ERFURTH, I., S. JOLIVET, N. FROGER, O. CATRICE $\&$ M. NOVATCHKOVA. 2008. Mutations in AtPS1 (Arabidopsis thaliana Parallel Spindle 1) lead to the production of diploid pollen grains. PLoS Genet 4: e1000274. doi:10.1371/journal.pgen.1000274.

DERNBURG, A. F., K. MCDONALD, G. MOULDER, R. BARSTEAD, M. DRESSER \& A. M. VILLENEUVE. 1998. Meiotic recombination in $C$. elegans initiates by a conserved mechanism and is dispensable for homologous chromosome synapsis. Cell 94: 387-398.

DE STORME, N. \& D. GEELEN. 2011. The Arabidopsis mutant jason produces unreduced FDR male gametes through a parallel/fused spindle mechanism in meiosis II. Plant Physiol. 155: 1403-1415.

DE WET, J. M. J. 1980. Origins of polyploids. In: LEWIS, 
W. H. (ed.), Polyploidy, Biological Relevance, pp. 3-16. Plenum Press, New York.

ELÍAS G, M. SARTOR \& V. G. SOLÍS NEFFA. 2011. Patterns of cytotype variation of Turnera sidoides subsp. pinnatifida (Turneraceae) in mountain ranges of central Argentina. J. Plant Res. 124: 25-34.

FERNÁNDEZ, A. 1973. El ácido láctico como fijador cromosómico. Bol. Soc. Argent. Bot. 15: 287-290.

FERNÁNDEZ, A. 1987. Estudios cromosómicos en Turnera y Piriqueta (Turneraceae). Bonplandia 6: $1-21$.

GIANSANTI, M. G., R. M. FARKAS, S. BONACCORSI, D. L. LINDSLEY, B. T. WAKIMOTO, M. T. FULLER \& M. GATTI. 2004. Genetic dissection of meiotic cytokinesis in Drosophila males. Mol. Biol. of the Cell 15: 2509-2522.

GRANT, V. 1989. Especiación vegetal. Ed. Limusa. Méjico.

GRELON, M., D. VEZON, G. GENDROT \& G. PELLETIER. 2001. AtSPO11-1 is necessary for efficient meiotic recombination in plants. Embo $J$. 20: 589-600.

HARLAN, J. R. \& J. M. J. DE WET. 1975. En Ö. WINGE and a prayer: the origins of polyploidy. Bot. Rev. 41: 361-390.

HERMSEM, J. G. T. 1984. Mechanisms and genetic implications of $2 n$ gamete formation. Iowa State J. Res. 58: 421-434.

JACKSON, R. C. \& J. CASEY. 1980. Cytogenetics of polyploids. In: LEWIS, W. H. (ed.), Polyploidy Biological Relevance, pp. 17-44. Plenum Press, New York.

JONGEDIJK, E. 1985. The pattern of megasporogenesis and megagametogenesis in diploid Solanum species hybrids; its relevance to the origin of $2 n$ eggs and the induction of apomixis. Euphytica 34: 599-611.

JONGEDIJK, E. \& M. S. RAMANNA. 1988. Synaptic mutants in potato, Solanum tuberosum L. I. Expression and identity of genes for desynapsis. Genome 30: 664-670.

KARPECHENKO, G. D. 1927. The production of polyploid gametes in hybrids. Hereditas 9: 349-368.

KAUL, M. L. H. \& T. G. K. MURTHY. 1985. Mutant genes affecting higher plant meiosis. Theor. Appl. Genet. 70: 449-466.

KHAN, N., R. BARBA GONZALEZ, M. S. RAMANNA, P. ARENS, R. G. F. VISSER \& J. M. VAN TUYL. 2010. Relevance of unilateral and bilateral sexual polyploidization in relation to intergenomic recombination and introgression in Lilium species hybrids. Euphytica 171: 157-173.

KODURU, P. R. K. \& M. K. RAO. 1981. Cytogenetics of synaptic mutants in higher plants. Theor. Appl. Genet. 59: 197-214.

KOVALSKY, I. E. 2012. Origen y establecimiento de poliploides en Turnera sidoides L. (Turneraceae). Tesis Doctoral, Universidad Nacional de Córdoba.

KOVALSKY, I. E. \& V. G. SOLÍS NEFFA. 2012. Evidence of $2 n$ microspore production in a natural diploid population of Turnera sidoides subsp. carnea and its relevance in the evolution of the $T$. sidoides (Turneraceae) autopolyploid complex. J. Plant. Res. 125: 725-734. DOI 10.1007/s10265-012-0493-7.

LEW, D. J. \& D. J. BURKET. 2003. The spindle assembly and spindle position checkpoints. Annu. Rev. Genet. 37: 251-282.

LIM, K. B., M. S. RAMANNA, J. H. DE JONG, E. JACOBSEN \& J. M. VAN TUYL. 2001. Indeterminate meiotic restitution (IMR): a novel type of meiotic nuclear restitution mechanism detected in interspecific lily hybrids by GISH. Theor. Appl. Genet. 103: 219-230.

LYRENE P. M., N. VORSA \& J. R. BALLINGTON. 2003. Polyploidy and sexual polyploidization in the genus Vaccinium. Euphytica 133: 27-36.

MASON A. S., M. N. NELSON, G. YAN \& W. A. COWLING. 2011. Production of viable male unreduced gametes in Brassica interespecific hybrids is genotype specific and stimulated by cold temperatures. BMC Plant. Biol. 11: 1-13.

MENDIBURU, A. O. \& S. J. PELOQUIN. 1977. The significance of $2 n$ gametes in potato breeding. Theor. Appl. Genet. 49: 53-61.

MUSACCHIO, A. \& K. G. HARDWICK. 2002. The spindle checkpoint: Structural insights into dynamic signaling. Nat. Rev.-Mol. Cell Biol. 3: 731-741.

NACRY, P., U. MAYER \& G. JURGENS. 2000. Genetic dissection of cytokinesis. Plant Mol. Biol. 43: 719-733.

PANSERI, A. F., J. G. SEIJO \& V. G. SOLÍS NEFFA. 2008. Análisis de la producción y frecuencia de microsporas no reducidas en diploides de Turnera sidoides (Turneraceae). Bol. Soc. Argent. Bot. 43: 95-101.

PARROT, W. A. \& R. R. SMITH. 1984. Production of $2 n$ pollen in red clover. Crop Sci. 24: 469-472.

PARROTT, W. A. \& R. E. HANNEMAN Jr. 1988. Megasporogenesis in normal and a synaptic-mutant (sy-2) of Solanum commersonii Dun. Genome 30: 536-539.

PITTENGER, T. H. \& E. F. FROLIK. 1951. Temporary mounts for pollen abortion determinations. Stain Technol. 26: 181-184.

QU, L. \& N. VORSA. 1999. Desynapsis and spindle abnormalities leading to $2 n$ pollen formation in Vaccinium darrowii. Genome 42: 35-40.

RAMANNA, M. S. 1979. A re-examination of the mechanisms of $2 n$ gametes formation in potato and its implications for breeding. Euphytica 28: 537-561. 
RAMANNA, M. S. 1983. First division restitution gametes through fertile desynaptic mutants of potato. Euphytica 32: 337-350.

RAMANNA, M. S. 1992. The use of $2 n$-gametes in breeding polysomic polyploid species: Some achievements and perspectives. In: BARBAGONZALEZ, R., K. B. LIM, M. S. RAMANNA $\&$ J. M. VAN TUYL. 2005. Use of $2 n$ Gametes for inducing intergenomic recombination in Lily hybrids. Acta Hort. 673: 161-166.

RAMANNA, M. S. \& E. JACOBSEN. 2003. Relevance of sexual polyploidization for crop improvement - A review. Euphytica 133: 3-18.

RAMSEY, J. \& D. W. SCHEMSKE. 1998. Pathways, mechanisms, and rates of polyploid formation in flowering plants. Annu. Rev. Ecol. Evol. S. 29: 467-501.

SINGH, A. K., J. P. MOSS \& J. SMART. 1990. En: BRETAGNOLLE, F. \& J.D. THOMPSON. 1995. Gametes with the somatic chromosome number: mechanisms of their formation and role in the evolution of autopolyploid plants. New Phytol. 129: $1-22$.

SOLÍS NEFFA, V. G. 2000. Estudios biosistemáticos en el complejo Turnera sidoides L. (Turneraceae, Leiocarpae). Tesis Doctoral, Universidad Nacional de Córdoba.

SOLÍS NEFFA, V. G. \& A. FERNÁNDEZ. 2001. Cytogeography of the Turnera sidoides L. complex (Turneraceae, Leiocarpae). Bot. J. Linn. Soc. 137: 189-196.

SOLÍS NEFFA, V. G., A. F. PANSERI, W. REYNOSO \& J. G. SEIJO. 2004. Variación en el color de flores y números cromosómicos en el Noroeste del área de distribución de Turnera sidoides (Turneraceae). Bonplandia 13: 117-128.

STEBBINS, G. L. 1971. Chromosomal evolution in higher plants. Addison-Wesley Press, London.

TASCHETTO, O. M. \& M. S. PAGLIARINI. 2003. Occurrence of $2 n$ and jumbo pollen in the Brazilian ginseng (Pfaffia glomerata and P. tuberosa). Euphytica 133: 139-145.

TOTH, A., K. P. RABITSCH, M. GALOVA, A. SCHLEIFFER, S. B. C. BUONOMO \& K. NASMYTH. 2000. Functional genomics identifies monopolin: a kinetochore protein required for segregation of homologs during meiosis I. Cell 103: 1155-1168.

THOMPSON J. D. \& R. LUMARET. 1992. The evolutionary dynamics of polyploid plants: origins, establishment and persistence. Trends Ecol. Evol. 7: 302-307.
VEILLEUX, R. E. 1985. Diploid and polyploid gametes in crop plants: mechanisms of formation and utilization in plant breeding. Plant Breed. Rev. 3: 253-288.

WATANABE, Y. \& P. NURSE. 1999. Cohesin Rec8 is required for reductional chromosome segregation at meiosis. Nature 400: 461-464.

WATANABE, Y. \& S. YOKOBAYASHI. 2005. The kinetochore protein Moal enables cohesionmediated monopolar attachment at meiosis I. Cell 123: 803-817.

WERNER, J. E. \& S. J. PELOQUIN. 1991a. Occurrence and mechanism of $2 n$ egg formation in $2 \mathrm{x}$ potato. Genome 34: 975-982.

WERNER, J. E. \& S. J. PELOQUIN. 1991b. Yield and tuber characteristics of $4 \mathrm{x}$ progeny from $2 \mathrm{x}^{\prime} 2 \mathrm{x}$ crosses. Potato Res. 34: 261-267.

WERNER, J. E. \& S. J. PELOQUIN. 1991c. Significance of allelic diversity and $2 n$ gametes for approaching maximum heterozygosity in $4 x$ potatoes. Euphytica 58: 21-29.

XU, S. \& Y. DONG. 1992. Fertility and meiotic mechanisms of hybrids between chromosome autoduplication tetraploid wheats and Aegilops species. Genome 35: 379-384.

XU, S. J. \& L. R. JOPPA. 1995. Mechanisms and inheritance of first division restitution in hybrids of wheat, rye, and Aegilops squarrosa. Genome 38: 607-615.

XU L. Q., Q. L. ZHANG \& Z. R. LUO. 2008. Occurrence and cytological mechanism of $2 n$ pollen formation in Chinese Diospyros spp. (Ebenaceae) staminate germplasm. J. Hortic. Sci. Biotech. 83: 668-672.

XUE, Z., L. PING \& L. MENGJUN. 2011. Cytological mechanism of $2 n$ pollen formation in Chinese jujube (Ziziphus jujuba Mill. 'Linglingzao'). Euphytica 182: 231-238.

ZHANG, Z. \& X. KANG. 2010. Cytological characteristics of numerically unreduced pollen production in Populus tomentosa Carr. Euphytica 173: $151-159$.

ZHOU, S., M. S. RAMANNA, R. G. F. VISSER \& J. M. VAN TUYL. 2008. Genome composition of triploid lily cultivars derived from sexual polyploidization of Longiflorum x Asiatic hybrids (Lilium). Euphytica 160: 207-215.

Recibido el 17 de octubre de 2013, aceptado el 12 de noviembre de 2013. 